\title{
Bilateral ureteral replacement using ileum in bilateral refractory ureteral stricture with renal insufficiency: a case report and review of literature
}

\author{
Jae Joon Park ${ }^{1 \#}$, Sangchul Yun ${ }^{2 \#}$, Jae Heon Kim ${ }^{1 \#}$ \\ ${ }^{1}$ Department of Urology, ${ }^{2}$ Department of Surgery, Soonchunhyang University Seoul Hospital, Soonchunhyang University Medical College, Seoul, \\ Republic of Korea \\ \#These authors contributed equally to this work. \\ Correspondence to: Jae Heon Kim, MD, PhD. Department of Urology, Soonchunhyang University Seoul Hospital, Soonchunhyang University Medical \\ College, 59, Daesagwan-ro, Yongsan-gu, Seoul, Republic of Korea. Email: piacekjh@hanmail.net.
}

\begin{abstract}
Although several studies have reported the efficacy and safety of ileal ureter in refractory ureteral strictures, reports on its long-term outcomes are still insufficient. In this case report, we present an excellent long-term outcome over three years without complication of bilateral ureteral replacement with ileum using ' 7 ' shaped configuration in a woman after undergoing radical hysterectomy and radiation therapy who had renal insufficiency due to ureteral stricture. This study gave us a useful information about the feasibility and safety of bilateral ileal ureter in patients with ureteral strictures and renal insufficiency.
\end{abstract}

Keywords: Bilateral ureteral replacement using ileum; ileal ureter; ureteral stricture; case report

Submitted Oct 23, 2019. Accepted for publication Mar 11, 2020.

doi: $10.21037 /$ tau.2020.03.32

View this article at: http://dx.doi.org/10.21037/tau.2020.03.32

\section{Introduction}

Iatrogenic injuries following ureteroscopic procedures or gynecologic malignant cancer surgeries have become the most common causes of ureteral obstruction $(1,2)$. Moreover, radiation therapy following radical surgery is one of common reason for refractory ureteral stricture to minimal invasive techniques including utereroscopic and percutaneous procedures (1-3). Among surgical treatments for long ureteral defect or refractory ureteral stricture especially in the upper ureter, ileal interposition for ureteral defect is useful for preserving renal function and escaping from external device including percutaneous nephrostomy tube $(3,4)$. Several studies have shown the efficacy of ureteral replacement using ileum of unilateral or bilateral ureteral strictures. However, long term results are still needed to establish this procedure as a standard treatment for refractory ureteral stricture. Moreover, recurrent UTI and metabolic acidosis could be common complications of ileal interposition for ureteral stricture (1-3). Recently, we experienced a case of bilateral refractory ureteral stricture with renal insufficiency treated with bilateral ureteral replacement using ileum. The patient showed an excellent long-term clinical outcome. Here we report this case with detailed surgical procedures and images.

We present the following case in accordance with the CARE reporting checklist (available at http://dx.doi. org/10.21037/tau.2020.03.32).

\section{Case presentation}

\section{Patient information and clinical findings}

A 41-year-old woman visited our urology department with bilateral refractory ureteral strictures with percutaneous nephrostomy (PCN) state. She has a history of radical hysterectomy with extended pelvic lymph node dissection due to advanced cervical cancer. She had adjuvant chemotherapy and radiation therapy 3 years before the visit. She had been treated with percutaneous ureteral dilation using ureteral ballooning which had failed due to none penetration of ureteral stent through those ureters. She had 


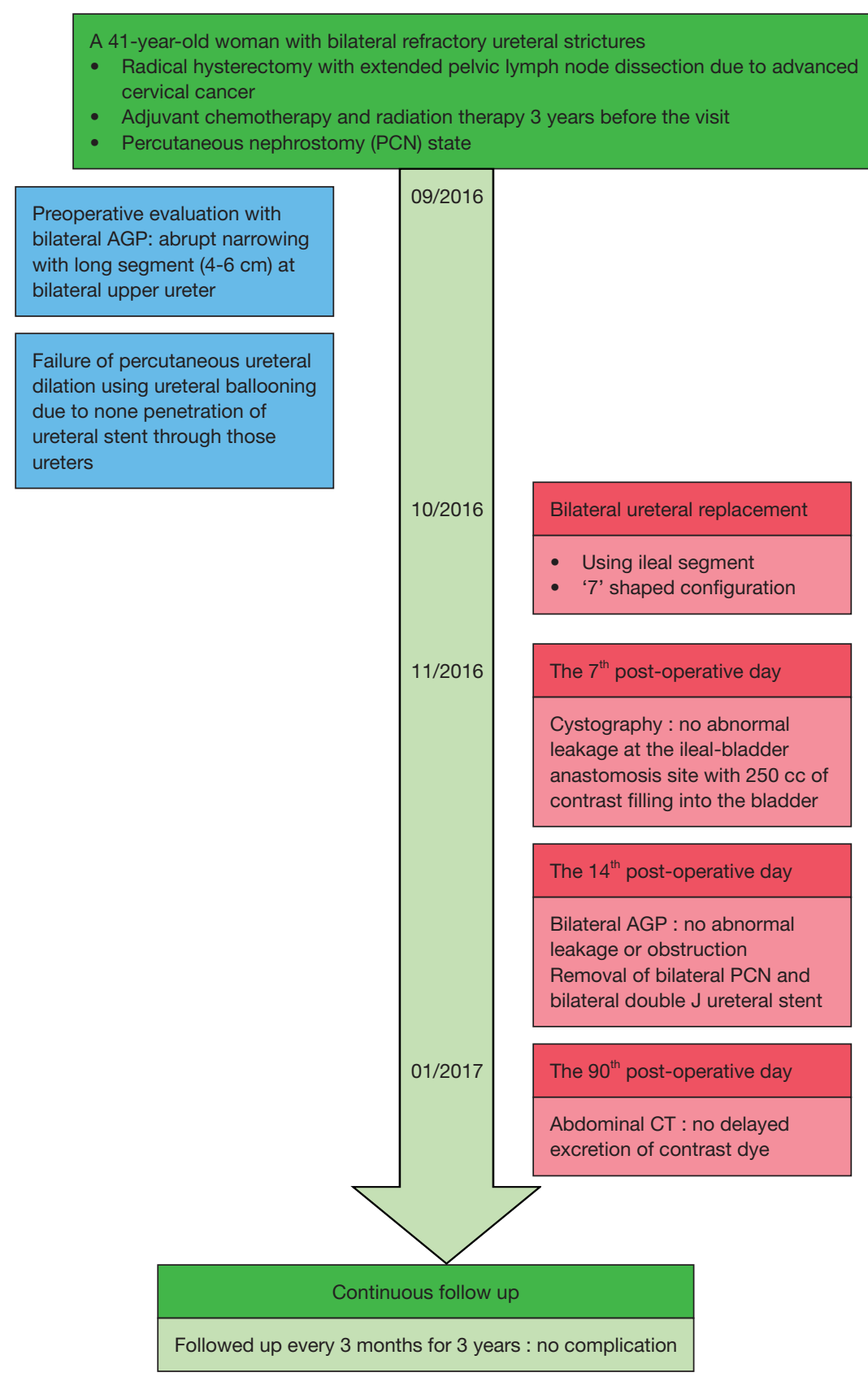

Figure 1 Patient timeline. PCN, percutaneous nephrostomy; AGP, anterograde pyelography; CT, computed tomography.

been indwelling bilateral PCN tubes for more than two years due to renal insufficiency (Figure 1).

\section{Diagnostic assessment}

Although her serum creatinine level was abnormal at 2.1 $\mathrm{ng} / \mathrm{mL}$, we decided to perform a reconstructive surgery using ileum considering her young age and that her other lab results were normal. Before surgical treatment, she underwent preoperative evaluation with bilateral Anterograde pyelography (AGP) which showed abrupt narrowing with long segment (4-6 cm) at bilateral upper ureter (Figure 2). 

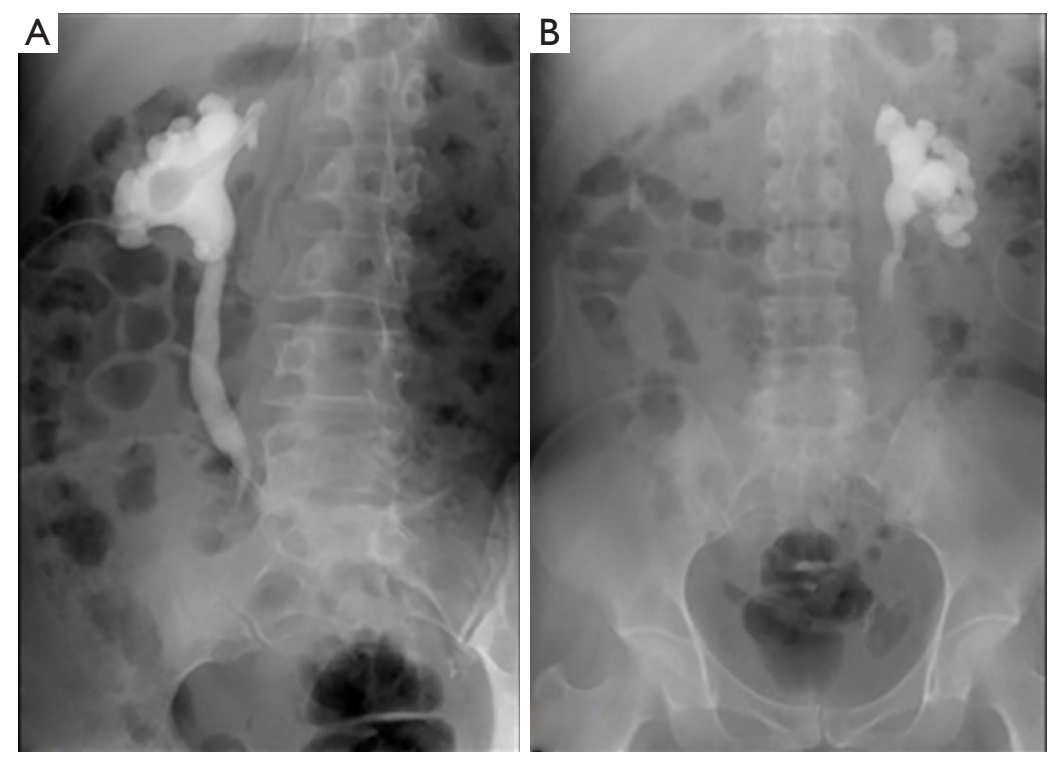

Figure 2 Anterograde pyelography before surgery. Ureteral strictures were confirmed with long segment at L4-5 level (A) and L3-4 level (B).
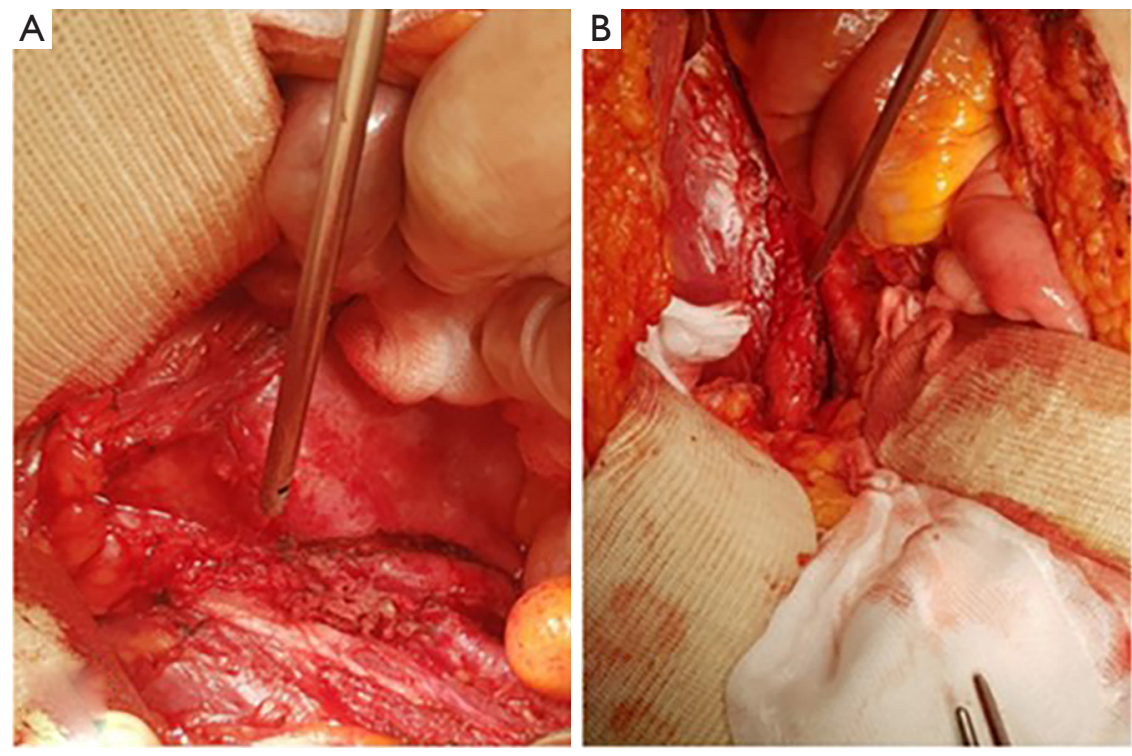

Figure 3 Intraoperative gross findings. Ureteral strictures were confirmed without peristalsis above the aorta bifurcation level at right side (A) and left side (B).

\section{Surgical methods and procedures}

Considering bilateral long segment, we performed bilateral ureteral replacement with ileum using ' 7 ' shaped configuration. The patient was in supine position under general anesthesia. After lower midline abdominal incision, bilateral ureteral dissection was done carefully to avoid any bowel injury because there were moderate to severe bowel adhesions. Finally, we could identify bilateral ureters with long stricture segments which had changed into firm tissues without any peristalsis (Figure 3). After dissecting and resecting bilateral upper segmented ureteral stricture sites, harvesting ileal segment for ileal ureter replacement was performed. Ileum was resected with $50 \mathrm{~cm}$ length starting 

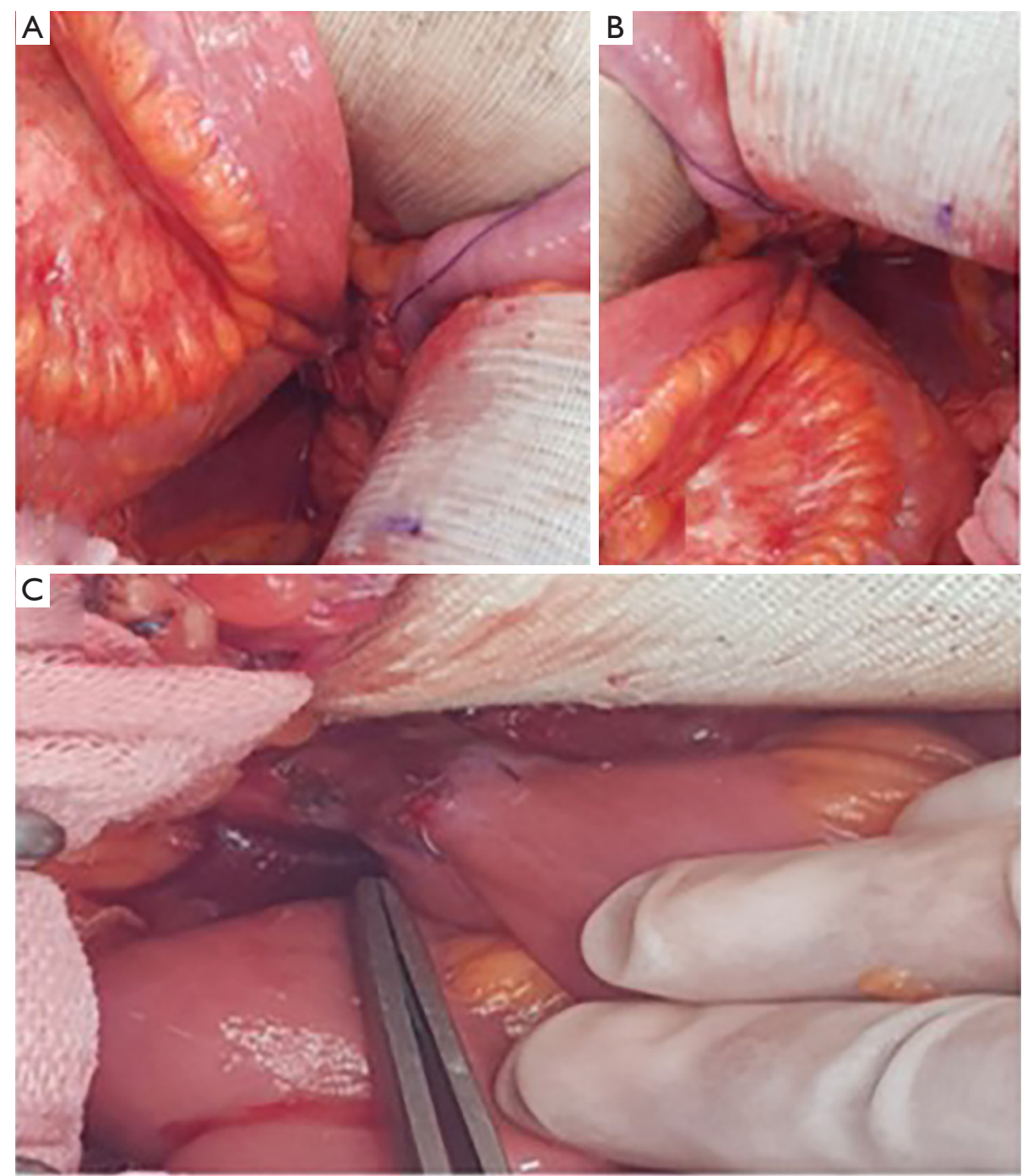

Figure 4 Surgical findings for anastomosis. Ileal-ureter anastomosis at right side (A), Ileal-ureter anastomosis at left side (B) and ilealbladder anastomosis $(\mathrm{C})$.

$30 \mathrm{~cm}$ proximal to the ileocecal valve. Remnant small bowels were hand-sewn anastomosed with end-to-end fashion and reinforced with Lambert sutures. Divided mesentery was closed carefully to prevent bowel hernia. Care was taken not to tighten the pedicle of harvested ileal segment. Harvested ileal segment have to be placed under the closed mesentery. Bilateral double $\mathrm{J}$ stents positioned in ureters were inserted into the harvested proximal ileal segment. Both ureters were anastomosed with the proximal part of the harvested ileal segment which was incised with the diameter of the ureter (Figures $4 A, B$ ). The superior portion of the urinary bladder was incised and anastomosed with the distal end of the harvested ileal segment (Figure 4C).

\section{Follow-up and outcomes}

On the $7^{\text {th }}$ post-operative day, cystography was performed. It which showed no abnormal leakage at the ileal-bladder anastomosis site with $250 \mathrm{cc}$ of contrast filling into the bladder (Figure $5 A$ ). On the $14^{\text {th }}$ post-operative day, bilateral AGP was done. It showed no abnormal leakage or obstruction (Figure 5B). After removal of bilateral PCN and bilateral double J ureteral stent, she was discharged. On the $90^{\text {th }}$ post-operative day, follow up abdominal computed tomography showed no delayed excretion of contrast dye with similar post hydronephrosis change compared to preoperative computed tomography image (Figure 6). She 

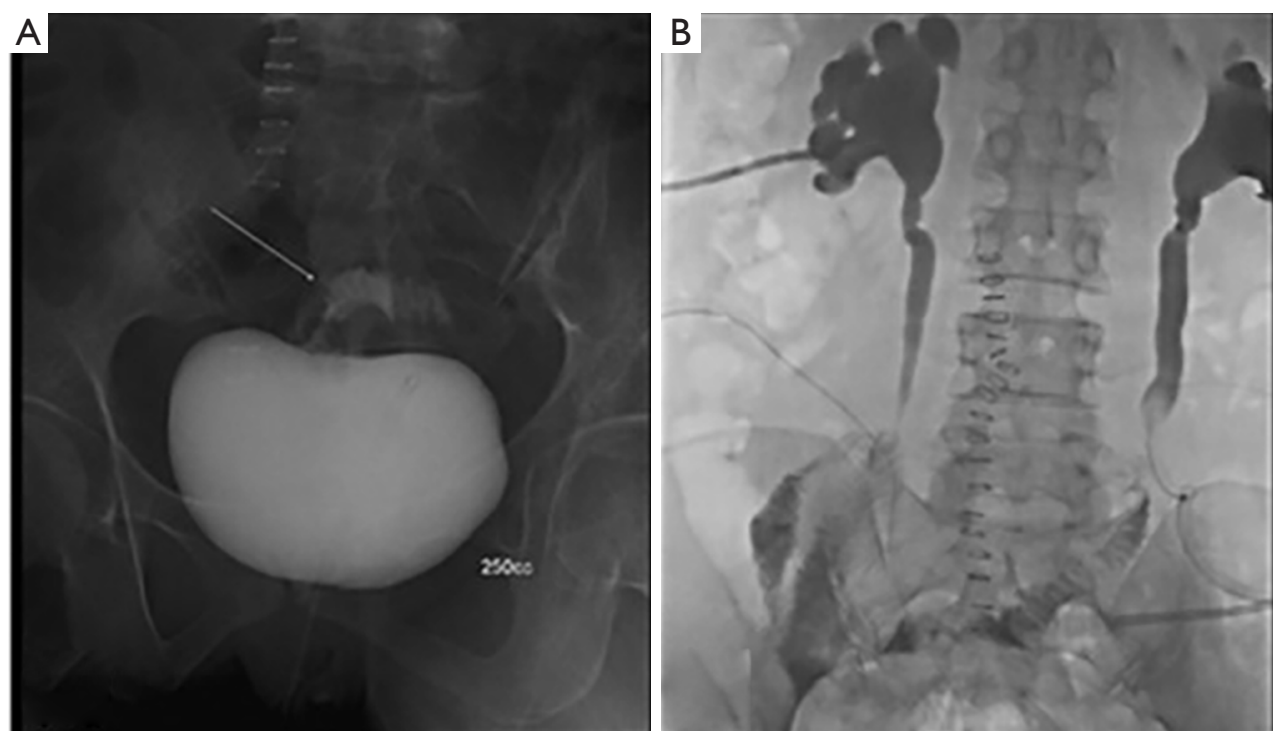

Figure 5 Post-operative images including cystography and anterograde pyelography. Post-operative cystography at 7 th post-operative day showing no abnormal findings (A) and post-operative anterograde pyelography showing no leakage without obstruction (B).

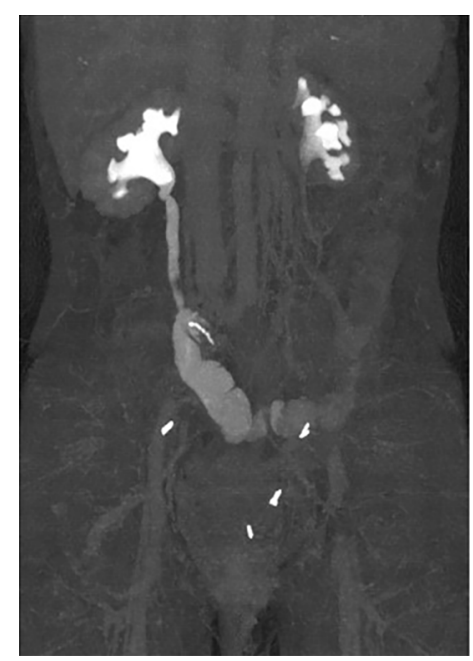

Figure 6 Post-operative images with computed tomography. Postoperative computed tomography at 90ths post-operative days showed no leakage without obstruction.

was followed up every 3 months for 3 years, showing no complication.

\section{Patient perspective}

Before this treatment, I was told that my urine flow was blocked. Since then, I have lived with a catheter in my kidney. It was too uncomfortable and painful for daily life.
But since the surgery, I have not needed catheter and have been able to live my normal life. There is nothing wrong with regular inspections so far. I am so happy with this point and grateful.

\section{Discussion}

Reconstructive procedures are required for ureteral obstructions caused by various reasons including primary retroperitoneal fibrosis, iatrogenic ureteral trauma, postradiotherapy fibrosis, and recurrent or refractory ureteral stenosis to avoid damage to affected kidney $(1,3)$. Minimal invasive procedures including antegrade or retrograde ureteral dilation with ureteral stenting could not reach a satisfactory clinical outcome. For those refractory ureteral strictures, ureteral replacement with ileum could be an ideal treatment option. However, its long-term efficacy and safety still need to be validated. Furthermore, the main advantage of this procedure is that patients do not need external device such as nephrostomy tube after this procedure. The main purpose of this study was to report the long-term favorable outcome with safety in a woman with refractory bilateral ureteral strictures and renal insufficiency.

Ureteral replacement using ileum with ileal-ureter interposition was first described for ureteral obstruction in a patient having tuberculosis (3). This operation had high mortality, morbidity, and complication rates in early 


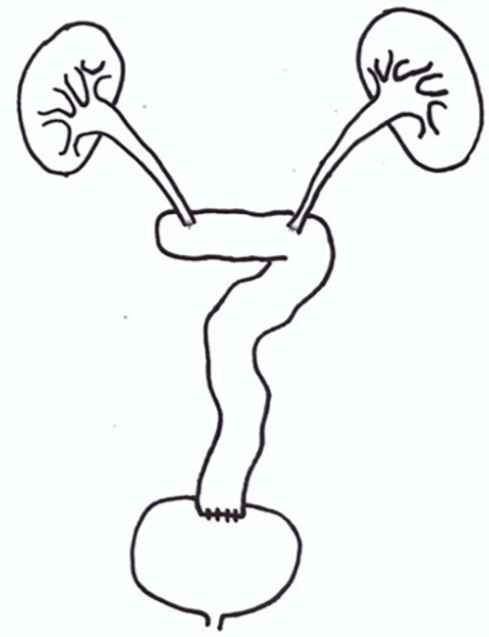

Figure 7 Surgical design with ' 7 ' shaped configuration. The length of harvested ileum was $50 \mathrm{~cm}$.

series. However, recent studies have shown that ureteral replacement using ileum has favorable outcomes $(1,3)$. The main etiology if ureteral stricture was urinary tuberculosis in the past. However, nowadays, the most common etiologies are iatrogenic injuries and post-operative change dues due to radical operation or post sequalae of radiation therapy $(1,2)$.

There are several configurations of ileal interposition for bilateral ureter defects established by many surgeons. These configurations are selected according to the length of the remaining normal ureter. An inverted ' 7 ' shaped configuration is method in which the ileum is placed in the form of number seven. It is appropriate for most bilateral ureter defects. A ' 7 ' shaped configuration is also appropriate for patients who have shorter length of the normal right ureter than the normal left ureter (3). Although urologists have tried to use as short length of ileum as possible for a variety of reasons, sometimes ' $\mathrm{Y}$ ' configuration and '11' configuration face challenges due to the length of mesentery, bowel status, and anatomical differences. The most important consideration when choosing a surgical procedure is obtaining tension-free watertight ureterileum anastomosis (3). In the present case, although the length of the normal right ureter was not shorter than the length of the normal left ureter, both ureters were still short. Considering that the right kidney had relatively more function than the left kidney, the ' 7 ' shaped configuration was used (Figure 7). In addition, the original position of the harvested ileal segment was close to the shaped of ' 11 ' number seven. Moreover, the mesentery and associated organ tend to be maintained in their original position by anatomical mechanisms such as peritoneal reflection, Toldt's fascia, and vascular connectivity (5). In this case, if we applied an inverted ' 7 ' configuration, the reconstructed ureteral structure might be at risk of twisting or tensioning when the harvested ileal segment returns to its original position.

Colon can be chosen as bowel interposed. However, ileum is preferred because of its mobility, blood supply, and small diameter (2). Long-term complications caused by interposition of bowel segment in urinary tract, such as acid-base imbalance, electrolyte imbalance, malabsorption of vitamins, cholelithiasis, and nephrolithiasis, depend on the kind and length of bowel harvested (6). Metabolic acidosis is a common complication of bowel interposition surgery. This complication can occur when colon or ileum is used for bowel interposition. However, the possibility of metabolic imbalance is lower when colon is used than when ileum is used. This metabolic acidosis can be treated by using oral sodium bicarbonate or sodium citrate plus citric acid (6). In the present case, the patient also experienced mild metabolic acidosis which was generally managed by intermittent administration of oral sodium bicarbonate according to our prior education before discharge.

After bowel interposition surgery, hypokalemia caused by electrolyte imbalance can occur as a longterm complication. Using colon is more vulnerable to hypokalemia than using ileum because if high potassium solution is in contact with bowel mucosa, the ileum can reabsorb the ion better than the colon (6). The patient in the present case also showed mild hypokalemia at 30 days after operation. It was managed well by intermittent administration of oral potassium chloride.

In terms of absorption of vitamin B12, using colon can be better than using ileum. Due to anatomical and physiological reasons, vitamin B12 absorption is intact when colon is used. However, B12 malabsorption can occur when ileum is used (6). Furthermore, if the length of harvested bowel is longer than $60 \mathrm{~cm}$ or the length of sparing terminal ileum is shorter than $15 \mathrm{~cm}$, the risk of B12 malabsorption may be higher $(6,7)$. In our patient, there was no abnormal finding of serum vitamin B12. From three months later after surgery, the patient showed stable acid balance state and electrolyte that she did not need any administration of sodium bicarbonate or oral potassium chloride.

There have been several studies about ureteral injury due to ileal interposition. These studies have shown frequencies of indications and complications of ureteral replacement 
operation. Common indications for ureteral replacement surgery are iatrogenic ureteral injury (such as surgery or endoscopic procedure), urinary tuberculosis, and postradiotherapy fibrosis. In these studies, the configuration of ileal segment for bilateral ureteral defects varies, including inverted seven shape, seven shape, eleven shape, $U$ shape, and $\mathrm{Y}$ shape. In these studies, the average length of follow- up period was 34.7 months. Complications of this surgery can be divided into short-term complications and longterm complications. Common short-term complications are leakage of anastomosis site, wound infection, and urinary tract infection. Common long-term complications are recurrent urinary tract infection, metabolic acidosis, and stricture of anastomosis site (Table 1) (1-3,8).

Table 1 Previous studies about ureteral replacement using ileum in refractory ureteral strictures

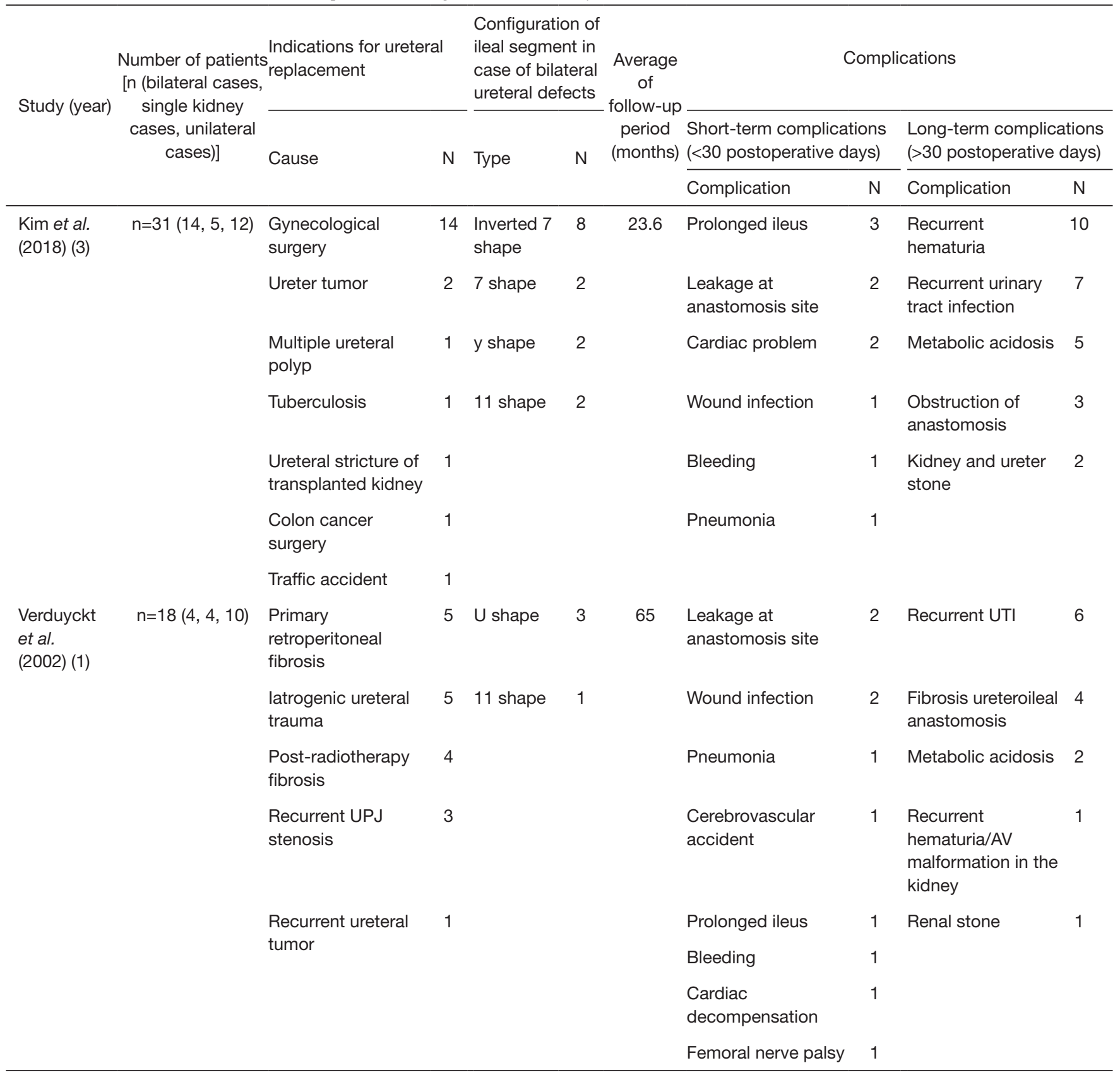

Table 1 (continued) 
Table 1 (continued)

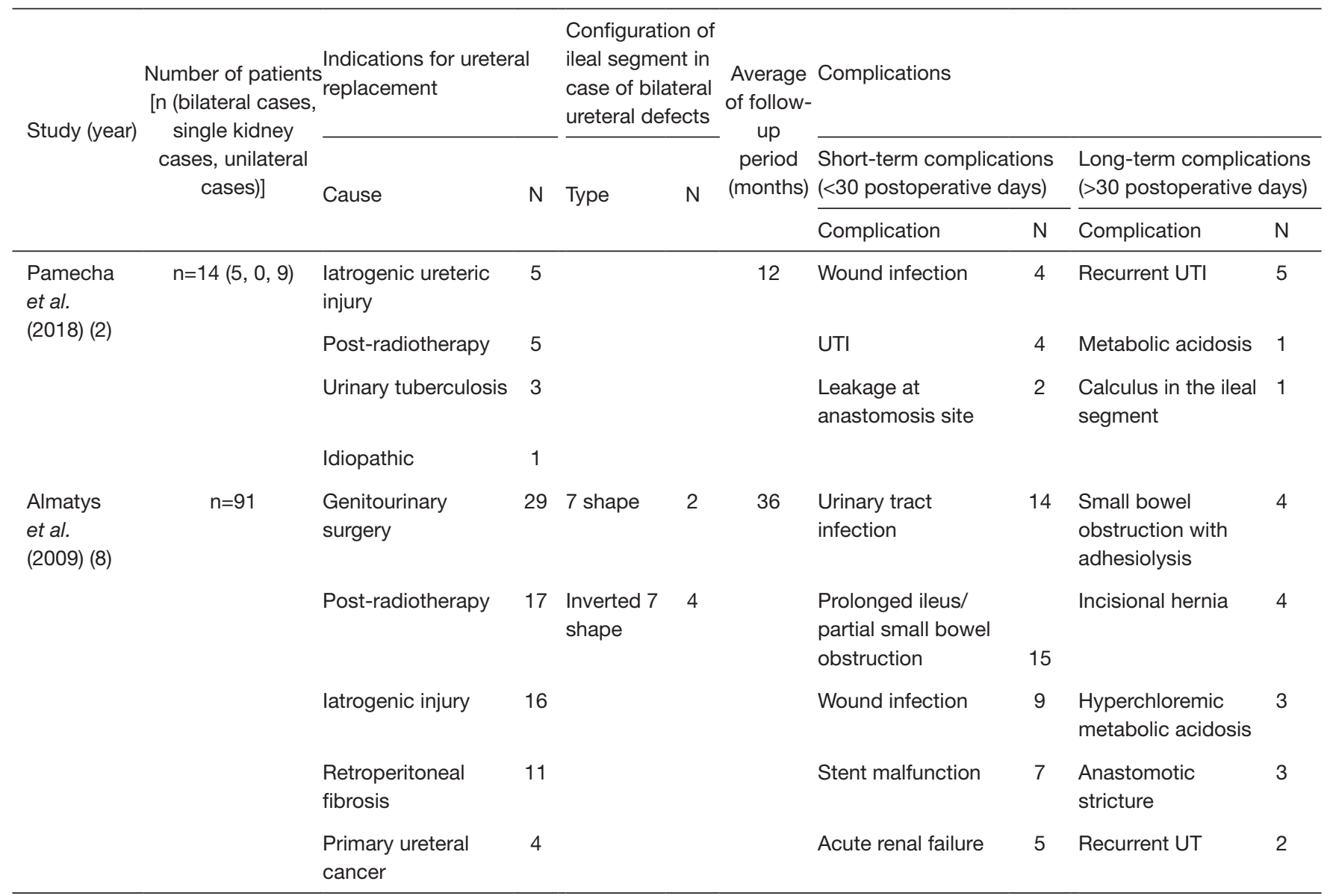

To date, there has been no clinical evidence about performing ureteral replacement using ileum in patients with renal insufficiency. In fact, several studies have suggested that renal insufficiency is one of contraindications for ureteral replacement using ileum $(2,9)$. Moreover, radiation is one of contraindication due to its possibility of bowel adhesions, risk of fistula formation, and high reoperation rate $(2,9)$.

\section{Conclusions}

Although more studies are needed to suggest that ureteral replacement using ileum could be a standard treatment strategy for refractory ureteral strictures, the present case suggests that its long term clinical outcome is favorable and safe. Moreover, this study suggests a positive role of ureteral replacement using ileum even in patients with renal insufficiency and radiation therapy.

\section{Acknowledgments}

Funding: This work was supported by Soonchunhyang University Research Fund.

\section{Footnote}

Reporting Checklist: The authors have completed the CARE reporting checklist. Available at http://dx.doi.org/10.21037/ tau.2020.03.32

Conflicts of Interest: All authors have completed the ICMJE uniform disclosure form (available at http://dx.doi. org/10.21037/tau.2020.03.32). The authors have no conflicts of interest to declare.

Ethical Statement: The authors are accountable for all aspects of the work in ensuring that questions related 
to the accuracy or integrity of any part of the work are appropriately investigated and resolved. The patient has signature informed consent.

Open Access Statement: This is an Open Access article distributed in accordance with the Creative Commons Attribution-NonCommercial-NoDerivs 4.0 International License (CC BY-NC-ND 4.0), which permits the noncommercial replication and distribution of the article with the strict proviso that no changes or edits are made and the original work is properly cited (including links to both the formal publication through the relevant DOI and the license). See: https://creativecommons.org/licenses/by-nc-nd/4.0/.

\section{References}

1. Verduyckt FJ, Heesakkers JP, Debruyne FM. Long-Term Results of Ileum Interposition for Ureteral Obstruction. Eur Urol 2002;42:181-7.

2. Pamecha Y, Shelke U, Patil B, et al. Use of ileum for complex ureteric reconstruction: Assessment of long-term outcome, complications, and impact on renal function. Urol Ann 2018;10:369-74.

Cite this article as: Park JJ, Yun S, Kim JH. Bilateral ureteral replacement using ileum in bilateral refractory ureteral stricture with renal insufficiency: a case report and review of literature. Transl Androl Urol 2020;9(3):1466-1474. doi:10.21037/ tau.2020.03.32
3. Kim A, Nam W, Song SH, et al. Use of the Ileum for Ureteral Stricture and Obstruction in Bilateral, Unilateral, and Single-kidney Cases. Urology 2018;111:203-7.

4. Chung BI, Hamawy KJ, Zinman LN, et al. The use of bowel for ureteral replacement for complex ureteral reconstruction: long-term results. J Urol 2006;175:179-83.

5. Byrnes KG, Walsh D, Dockery P, et al. Anatomy of the mesentery: Current understanding and mechanisms of attachment. Semin Cell Dev Biol 2019;92:12-7.

6. Martini A, Villari D, Nicita G. Long-term complications arising from bowel interposition in the urinary tract. Int $\mathrm{J}$ Surg 2017;44:278-80.

7. Fujisawa M, Gotoh A, Nakamura I, et al. Long-term assessment of serum vitamin B12 concentrations in patients with various types of orthotopic intestinal neobladder. Urology 2000;56:236-40.

8. Armatys SA, Mellon MJ, Beck SD, et al. Use of Ileum as Ureteral Replacement in Urological Reconstruction. J Urol 2009;181:177-81.

9. Takeuchi M, Masumori N, Tsukamoto T. Ureteral Reconstruction With Bowel Segments: Experience With Eight Patients in a Single Institute. Korean J Urol 2014;55:742-9. 